\title{
EVOLUTION EQUATIONS OF CURVATURE TENSORS ALONG THE HYPERBOLIC GEOMETRIC FLOW
}

\author{
WEI-JUN LU
}

\begin{abstract}
AвSTRACT. We consider the hyperbolic geometric flow $\frac{\partial^{2}}{\partial t^{2}} g(t)=-2 R i c_{g(t)}$ introduced by Kong and Liu [KL]. When the Riemannian metric evolve, then so does its curvature. Using the techniques and ideas of S.Brendle $[\overline{\mathrm{Br}} \mid \mathrm{BS}]$, we derive evolution equations for the Levi-Civita connection and the curvature tensors along the hyperbolic geometric flow. The method and results are computed and written in global tensor form, different from the local normal coordinate method in [DKL1]. In addition, we further show that any solution to the hyperbolic geometric flow that develops a singularity in finite time has unbounded Ricci curvature.
\end{abstract}

\section{INTRODUCTION}

Geometric flows are important in many fields of mathematics and physics. A geometric flow is an evolution of a geometric structure under a differential equation related to a functional on a manifold, usually associated with some curvature. The most popular geometric

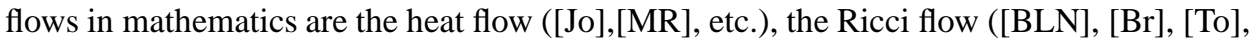
etc.), the mean curvature flow $([\overline{\mathrm{Zh}}])$ and the Yamabe flow ([Br1, $\mathrm{Br} 2, \mathrm{Ch} 1, \mathrm{SS}, \mathrm{Re}])$.

The subject of Hamilton's Ricci flow $|\mathrm{Ha}|: \frac{\partial}{\partial t} g(t)=-2 R i c_{g(t)}$ lies in the more general field of geometric flows, which in turn lies in the even more general field of geometric analysis. In Ricci flow we see the unity of geometry and analysis. As a fully nonlinear system of parabolic partial differential equations of second order ([De] $)$, the Ricci flow in many respects appears to be very natural equation.

Similarly, since the hyperbolic equation or system is one of the most natural models in the nature, we feel the hyperbolic geometric flow (for short, HGF): $\frac{\partial^{2}}{\partial t^{2}} g(t)=-2 R i c_{g(t)}$ introduced by Kong and Liu $[\mathrm{KL}]$ in the year 2007, is also a very natural tool. Note that the elliptic and parabolic partial differential equations have been successfully applied to differential geometry and physics. A natural and important question is if we can apply the well-developed theory of hyperbolic differential equations to solve problems in differential geometry and theoretical physics. HGF is helpful to understand the wave character of the metrics, wave phenomenon of the curvatures, the evolution of manifolds and their structures (see [KL, DKL1, Ko]). For more discussions for related hyperbolic flows and their applications to geometry and Einstein equations, we refer to [Li].

In geometry, singularity is more difficult to define, especially when the structure is governed by the hyperbolic system and the topology of the space is allowed to change. A good example is the singularity developed in general relativity. The major problem here is that one has very little understanding of the global behavior of nonlinear hyperbolic systems when the spatial dimension is greater than one. It is almost for sure that a break through will be accomplished in geometry if one knows this type of equation better (See

2000 Mathematics Subject Classification. 53C20, 53C44, 58J45.

Key words and phrases. hyperbolic geometric flow; evolution equations of curvature tensors . 
[SY]). HGF equation is quite difficult to solve in all generality unlike the nonlinear elliptic problems with a well developed regularity theory. Although the short time existence of solutions is guaranteed by hyperbolic nature of the equations, their (long time) convergence to canonical geometric structures is analyzed under various conditions. So far, providing results of this sort have been developed. Among them, one may find the related works in [DKL2, He, KLX, KLW].

The goal in this paper is to work out the evolution equations for Riemannian, Ricci and scalar curvature under the hyperbolic geometric flow. More precisely, we will concentrate in obtaining the global forms of the evolutions under HGF. As for the local forms of the evolutions, Dai-Kong-Liu [DKL1] have obtained the following results.

To state our results, it will be convenient to assume that $(M, g(t)), t \in(0, T)$, is a family of complete Riemannian manifolds evolving under hyperbolic geometric flow. In addition, 'Ric' and 'scal' denote the Ricci and scalar curvature of $(M, g(t))$, respectively.

Theorem 1.1. Under the hyperbolic geometric flow $\frac{\partial^{2}}{\partial t^{2}} g(t)_{i j}=-2 R i c_{i j}$, the curvature tensors satisfy the evolution equations

$$
\begin{aligned}
\frac{\partial^{2}}{\partial t^{2}} R_{i j k l}= & \Delta R_{i j k l}+2\left(B_{i j k l}-B_{i j l k}-B_{i l j k}+B_{i k j l}\right) \\
- & g^{p q}\left(R_{p j k l} R i c_{q i}+R_{i p k l} R i c_{q j}+R_{i j p l} R i c_{q k}+R_{i j k p} R i c_{q l}\right) \\
+ & 2 g_{p q}\left(\frac{\partial \Gamma_{i l}^{p}}{\partial t} \frac{\partial \Gamma_{j k}^{q}}{\partial t}-\frac{\partial \Gamma_{j l}^{p}}{\partial t} \frac{\partial \Gamma_{i k}^{q}}{\partial t}\right) \\
\frac{\partial^{2}}{\partial t^{2}} R i c_{i k} & =\Delta R i c_{i k}+2 g^{p r} g^{q s} R_{p i q k} R i c_{r s}-2 g^{p q} R i c_{p i} R i c_{q k} \\
& +2 g^{j l} g_{p q}\left(\frac{\partial \Gamma_{i l}^{p}}{\partial t} \frac{\partial \Gamma_{j k}^{q}}{\partial t}-\frac{\partial \Gamma_{j l}^{p}}{\partial t} \frac{\partial \Gamma_{i k}^{q}}{\partial t}\right) \\
& -2 g^{j p} g^{l q} \frac{\partial g_{p q}}{\partial t} \frac{\partial}{\partial t} R_{i j k l}+2 g^{j p} g^{r q} g^{s l} \frac{\partial g_{p q}}{\partial t} \frac{\partial g_{r s}}{\partial t} R_{i j k l}, \\
\frac{\partial^{2}}{\partial t^{2}} S c a l & =\Delta S c a l+2|R i c|^{2} \\
& +2 g^{i k} g^{j l} g_{p q}\left(\frac{\partial \Gamma_{i l}^{p}}{\partial t} \frac{\partial \Gamma_{j k}^{q}}{\partial t}-\frac{\partial \Gamma_{j l}^{p}}{\partial t} \frac{\partial \Gamma_{i k}^{q}}{\partial t}\right) \\
& -2 g^{i k} g^{j p} g^{l q} \frac{\partial g_{p q}}{\partial t} \frac{\partial}{\partial t} R_{i j k l} \\
& -2 g^{i p} g^{k q} \frac{\partial g_{p q}}{\partial t} \frac{\partial R i c_{i k}}{\partial t}+4 R i c_{i k} g^{i p} g^{r q} g^{s k} \frac{\partial g_{p q}}{\partial t} \frac{\partial g_{r s}}{\partial t}
\end{aligned}
$$

where $\left\{x^{i}\right\}$ is a local normal coordinates around a fixed point $p \in M, B_{i j k l}=g^{p r} g^{q s} R_{p i q j} R_{r k s l}$ and $\Delta$ is the Laplacian with respect to the evolving metric $g(t)$.

Analogous results for the Einstein's hyperbolic geometric flow (2.3) and the dissipative hyperbolic geometric flow (2.4) occur in ([|He]) and ([DKL2]), respectively.

Motivated by the techniques and ideas concerning the Ricci flow in S. Brendle's paper $[\mathrm{Br}, \overline{\mathrm{BS}}]$, we obtain the following main results with global forms under the HGF. 
EVOLUTION EQUATIONS OF CURVATURE TENSORS ALONG THE HYPERBOLIC GEOMETRIC FLOW 3

Theorem 1.2. Let $X, Y, Z, W$ be fixed vector fields on $M$. Then under the $H G F$ (2.1), we have

$$
\begin{aligned}
\frac{\partial^{2}}{\partial t^{2}} R(X, Y, Z, W) & =-\operatorname{Ric}_{g(t)}(R(X, Y) Z, W)+\operatorname{Ric}_{g(t)}(R(X, Y) W, Z) \\
& +\left(D_{X, Z}^{2} \operatorname{Ric}_{g(t)}\right)(Y, W)-\left(D_{X, W}^{2} \operatorname{Ric}_{g(t)}\right)(Y, Z) \\
& -\left(D_{Y, Z}^{2} \operatorname{Ric}_{g(t)}\right)(X, W)+\left(D_{Y, W}^{2} \operatorname{Ric}_{g(t)}\right)(X, Z) \\
& +2 \frac{\partial g(t)}{\partial t}\left(\left(D_{X} B\right)(Y, Z), W\right)-2 \frac{\partial g(t)}{\partial t}\left(\left(D_{Y} B\right)(X, Z), W\right) \\
& -2 g(t)(B(X, B(Y, Z)), W)+2 g(t)(B(Y, B(X, Z)), W),
\end{aligned}
$$

where $B(X, Y):=\frac{\partial}{\partial t}\left(D_{X} Y\right)$.

Theorem 1.3. Let $X, Y, Z, W$ be arbitrary fields on $M$. Then under the hyperbolic geometric flow (2.1), the curvature tensors satisfy the evolution equations

$$
\begin{aligned}
\frac{\partial^{2}}{\partial t^{2}} R(X, Y, Z, W)= & (\Delta R)(X, Y, Z, W)+Q(R)(X, Y, Z, W) \\
& -\operatorname{Ric}_{g(t)}\left(X, R_{Z, W} Y\right)+\operatorname{Ric}_{g(t)}\left(Y, R_{Z, W} X\right) \\
+ & R i c_{g(t)}\left(Z, R_{X, Y} W\right)-\operatorname{Ric}_{g(t)}\left(W, R_{X, Y} Z\right) \\
+ & 2 \frac{\partial g(t)}{\partial t}\left(\left(D_{X} B\right)(Y, Z), W\right)-2 \frac{\partial g(t)}{\partial t}\left(\left(D_{Y} B\right)(X, Z), W\right) \\
- & 2 g(t)(B(X, B(Y, Z)), W)+2 g(t)(B(Y, B(X, Z)), W),
\end{aligned}
$$

where $Q(R):=R^{2}+R^{\#}$ is a curvature tensor satisfying the first Bianchi identity, and given a local orthonormal basis $\left\{e_{1}, \ldots, e_{n}\right\}, R^{2}$ and $R^{\#}$ are difined by

$$
\begin{aligned}
& R^{2}(X, Y, Z, W):=\sum_{p, q=1}^{n} R\left(X, Y, e_{p}, e_{q}\right) R\left(e_{p}, e_{q}, Z, W\right), \\
& R^{\#}(X, Y, Z, W):=2 \sum_{p, q=1}^{n}\left|\begin{array}{ll}
R\left(X, e_{p}, Z, e_{q}\right) & R\left(X, e_{p}, W, e_{q}\right) \\
R\left(Y, e_{p}, Z, e_{q}\right) & R\left(Y, e_{p}, W, e_{q}\right)
\end{array}\right| .
\end{aligned}
$$

Theorem 1.4. Let $X, Y$ be arbitrary fields on $M$. Then under the hyperbolic geometric flow (2.1), the Ricci curvature tensor Ric $g_{g(t)}$ and scalar curvature scal ${ }_{g(t)}$ satisfy the evolution equations, respectively

$$
\begin{aligned}
\frac{\partial^{2}}{\partial t^{2}} \operatorname{Ric}_{g(t)}(X, Y) & =\left(\Delta R i c_{g(t)}\right)(X, Y)+2 \sum_{i, j=1}^{n} R\left(X, e_{i}, Y, e_{j}\right) R i c_{g(t)}\left(e_{i}, e_{j}\right) \\
& +2 \sum_{i=1}^{n}\left(\frac{\partial g(t)}{\partial t}\left(\left(D_{X} B\right)\left(e_{i}, Y\right), e_{i}\right)-\frac{\partial g(t)}{\partial t}\left(\left(D_{e_{i}} B\right)(X, Y), e_{i}\right)\right) \\
& -2 \sum_{i=1}^{n}\left(g(t)\left(B\left(X, B\left(e_{i}, Y\right)\right), e_{i}\right)-g(t)\left(B\left(e_{i}, B(X, Y)\right), e_{i}\right)\right), \\
\frac{\partial^{2}}{\partial t^{2}} S c a l_{g(t)}= & \Delta S c a l_{g(t)}+2\left|R i c_{g(t)}\right|^{2} \\
& \left.+2 \sum_{i, j=1}^{n}\left(\frac{\partial g(t)}{\partial t}\left(\left(D_{e_{j}} B\right)\left(e_{i}, e_{j}\right), e_{i}\right)\right)-\frac{\partial g(t)}{\partial t}\left(\left(D_{e_{i}} B\right)\left(e_{j}, e_{j}\right), e_{i}\right)\right) \\
- & 2 \sum_{i, j=1}^{n}\left(g(t)\left(B\left(e_{j}, B\left(e_{i}, e_{j}\right)\right), e_{i}\right)-g(t)\left(B\left(e_{i}, B\left(e_{j}, e_{j}\right)\right), e_{i}\right)\right),
\end{aligned}
$$


where $\left\{e_{1}, \ldots, e_{n}\right\}$ is a local orthonormal basis of $M$, and $|\operatorname{Ric}|^{2}=\sum_{i, j=1}^{n}\left(\operatorname{Ric}\left(e_{i}, e_{j}\right)\right)^{2}$.

The structure of the paper is as follows. In Section 2 we state the related concepts such as HGF, Einstein HGF and present some examples of specific solutions to the HGF. In Section 3 we give evolution equations for the Levi-Civita connection and prove the main results in the introduction. In Section 4 we consider Ricci curvature blow-up at finite-times singularities. Section 5 presents some expected problems.

\section{Hyperbolic GEOMETRIC FLOW}

Recall that Kong and Liu $[\mathrm{KL}]$ introduce a geometric flow-Hyperbolic geometric flow (HGF), which is difference from the Hamilton's Ricci flow, although these two flows share a common Ricci term $-2 R i c_{g(t)}$. The definition of HGF is as follows.

Definition 2.1. Let $M$ be a manifold. The hyperbolic geometric flow is the evolution

$$
\frac{\partial^{2}}{\partial t^{2}} g(t)=-2 R i c_{g(t)}
$$

for a one-parameter family of Riemannian metrics $g(t), t \in[0, T)$ on $M$. We say that $g(t)$ is a solution to the hyperbolic geometric flow if it satisfies (2.1).

Similar to the Ricci flow $\frac{\partial}{\partial t} g(t)=-2 R i c_{g(t)}$, the HGF equation (2.1) is a unnormalized evolution equation. In $[\overline{\mathrm{KL}}]$, Kong and Liu also consider the normalized version of hyperbolic geometric flow, which preserves the volume of the flow. Considering the HGF and the normalized HGF differ only by a change of scale in space $M$ and a change of time $t$, the normalized $H G F$ equation reads such form as

$$
\frac{\partial^{2}}{\partial t^{2}} g(t)=-2 R i c_{g(t)}+a(t) \frac{\partial}{\partial t} g(t)+b(t) g(t)
$$

where $a(t)$ and $b(t)$ are certain functions of $t$.

In order to further understand the relationship between the Einstein equation and the HGF, Kong and Liu also introduce a so-called Einstein's hyperbolic geometric flow.

Definition 2.2. Let $\mathbb{R} \times M$ be a space-time with the Lorentzian metric $d s^{2}=d t^{2}+$ $g_{i j}(x, t) d x^{i} d x^{j}$. Suppose the Einstein equations in the vacuum, which correspond to the metric $d s^{2}$, has the form

$$
\frac{\partial^{2}}{\partial t^{2}} g(t)_{i j}=-2 R i c_{i j}-\frac{1}{2} g^{p q} \frac{\partial g_{i j}}{\partial t} \frac{\partial g_{p q}}{\partial t}+g^{p q} \frac{\partial g_{i p}}{\partial t} \frac{\partial g_{j q}}{\partial t} .
$$

The equation (2.3) is called Einstein's hyperbolic geometric flow.

Motivated by the well-developed theory of the dissipative hyperbolic equations, DaiKong-Liu [DKL2] introduce a new geometric analytical tool-dissipative hyperbolic geometric flow defined by

$$
\begin{aligned}
\frac{\partial^{2}}{\partial t^{2}} g(t)_{i j} & =-2 R i c_{i j}+2 g^{p q} \frac{\partial g_{i p}}{\partial t} \frac{\partial g_{j q}}{\partial t}-\left(d+2 g^{p q} \frac{\partial g_{p q}}{\partial t}\right) \frac{\partial g_{i j}}{\partial t} \\
& +\frac{1}{n-1}\left(\left(g^{p q} \frac{\partial g_{p q}}{\partial t}\right)^{2}+\frac{\partial g^{p q}}{\partial t} \frac{\partial g_{p q}}{\partial t}\right) g_{i j},
\end{aligned}
$$

where $d$ is a positive constant. The reason that (2.4) is chosen as the equation form of dissipative hyperbolic geometric flow is that, in the case it possesses a simpler equation satisfied by the scalar curvature.

In order to get a feel for the HGF (2.1), we present some examples of specific solutions (cf. [KL, DKL1, DKL2, KLW, He]). 
Example 2.3. (i)(Trivial example) If the initial metric is Ricci flat, so that $R i c_{i j}=0$, then clearly the metric does not change under 2.1. Hence any Ricci flat metric $g(t)$ is a stationary solution to the hyperbolic geometric flow. This happens, for example, on the flat torus or on any K3-surface with a Calabi-Yau metric.

(ii)(Non-trivial example) A typical example of the Einstein metric is

$$
d s_{0}^{2}=\frac{1}{1-\kappa r^{2}} d r^{2}+r^{2} d \theta^{2}+r^{2} \sin ^{2} \theta d \varphi^{2},
$$

where $\kappa$ is a constant taking its value $-1,0$ or 1 . We can prove that the metric

$$
d s^{2}=\left(-2 \kappa t^{2}+c_{1} t+c_{2}\right) d s_{0}^{2}
$$

is a solution to the HGF (2.1) , where $c_{1}$ and $c_{2}$ are two constants.

Example 2.4. Consider the solution in conformal class with the following form

$$
g_{i j}(t, x)=\rho(t) g_{i j}(0, x) .
$$

Suppose that the initial metric $g_{i j}(0, x)$ is Einstein, that is, there exists some constant $\lambda$ such that

$$
\operatorname{Ric}_{i j}(0, x)=\lambda g_{i j}(0, x), \forall x \in M .
$$

Then (2.5) with $\rho(t)=-\lambda t^{2}+v t+1$ and a real number $v$ standing for the initial velocity, is a solution to the equation (2.1).

Example 2.5. For the Einstein's hyperbolic geometric flow (2.3), its exact solution has the following form

$$
d s^{2}=f(t, z) d z^{2}+\frac{h(t)}{F(t, z)}\left((d x-\mu(t, z) d y)^{2}+F^{2}(t, z) d y^{2}\right),
$$

where $f, h$ and $F$ are smooth functions with respect to variables. The $x$-invariance and $y$ invariance show that the model possesses the $z$-axial symmetry. In order to guarantee that the metric $g_{i j}$ is Riemannian, we assume $F(t, z)>0$ and $h(t) / F(t, z)>0$.

More interesting example comes from the Riemann surfaces with the initial asymptotic flat.

Example 2.6. On a surface, the HGF equation (2.1) can be simplified as the following equation for the special metric

$$
\frac{\partial^{2}}{\partial t^{2}} g_{i j}(t, x, y)=-S c a l g_{i j}(t, x, y)
$$

Because the Ricci curvature is given by

$$
\operatorname{Ric}_{i j}(t, x, y)=\frac{1}{2} S \operatorname{cal}(t, x, y) g_{i j}(t, x, y)
$$

where $S \mathrm{cal}=2 \mathrm{~K}$ is the scalar curvature function with the Gauss curvature $K$. Note that the metric for a surface can always be written (at least locally) as the following form

$$
g_{i j}(t, x, y)=u(t, x, y) \delta_{i j},
$$

where $u=u(t, x, y)>0$. Thus

This implies that (2.6) reads

$$
\text { Scal }=-\frac{1}{u} \Delta \log u
$$

$$
u_{t t}-\Delta \log u=0 .
$$


Define $f=\log u$, then (2.7) exchanges a quasilinear hyperbolic wave equation

$$
f_{t t}-e^{-f} \Delta u=-f_{t} \text {. }
$$

Consider the Cauchy problem for (2.8) with the following initial data

$$
t=0: f=\varepsilon f_{0}(x), f_{t}=\varepsilon f_{1}(x)
$$

and satisfying the slow decay condition

$$
\left|f_{0}(x)\right| \leq \frac{A}{(1+|x|)^{k}},\left|f_{1}(x)\right|=\frac{A}{(1+|x|)^{k+1}},
$$

where $\varepsilon>0$ is a suitably small parameter, and $f_{0}(x), f_{1}(x) \in C^{\infty}\left(\mathbb{R}^{2}\right) ; A, k$ are two suitable positive constants, $k>1$. Then there exist two positive constants $\delta$ and $\varepsilon_{0}$ such that for any fixed $\varepsilon \in\left[0, \varepsilon_{0}\right]$, the Cauchy problem $(2.8) \sim(2.10)$ has a unique $C^{\infty}$ solution on the interval $\left[0, T_{\varepsilon}\right]$ with $T_{\varepsilon}=\delta \varepsilon^{-4 / 3}$ (for detail, see [KLW]).

\section{Evolution OF THE CURVATURE TENSORS AND PROOFS OF MAIN RESULTS}

In this section, we derive evolution equations with global forms for the Levi-Cicvita connection and the curvature tensors along the HGF. We employ the techniques and ideas in studying evolution equations along the Ricci flow by S.Brendle (See $[\overline{\mathrm{Br}}]$ or $[\overline{\mathrm{BS}}]$ ).

From now on, we assume that $(M, g(t)), t \in(0, T)$, is a family of complete Riemannian manifolds evolving under HGF.

3.1. Evolution of the Levi-Civita connection. Let $X, Y$ be fixed vector fields on $M$ (that is, $X, Y$ are independent of $t$ ). We define

$$
A(X, Y):=\frac{\partial^{2}}{\partial t^{2}}\left(D_{X} Y\right), B(X, Y):=\frac{\partial}{\partial t}\left(D_{X} Y\right) .
$$

Observe that the difference of two connections is always a tensor, consequently, $A, B$ are tensors.

Proposition 3.1. Let $X, Y, Z$ be fixed vector fields on $M$. Then

$$
\begin{aligned}
g(t)(A(X, Y), Z) & =-\left(D_{X} \operatorname{Ric}_{g(t)}\right)(Y, Z)-\left(D_{Y} \operatorname{Ric}_{g(t)}\right)(X, Z) \\
& +\left(D_{Z} \operatorname{Ric}_{g(t)}\right)(X, Y)-2 \frac{\partial g(t)}{\partial t}(B(X, Y), Z) .
\end{aligned}
$$

Proof. For $g(t)\left(D_{X} Y, Z\right)$, we differentiate it twice with respect to $t$. This yields

$$
\frac{\partial^{2}}{\partial t^{2}}\left(g(t)\left(D_{X} Y, Z\right)\right)=\frac{\partial^{2} g(t)}{\partial t^{2}}\left(D_{X} Y, Z\right)+2 \frac{\partial g(t)}{\partial t}(B(X, Y), Z)+g(t)(A(X, Y), Z) .
$$

Since the Levi-Civita connection satisfies

$$
\begin{aligned}
2 g(t)\left(D_{X} Y, Z\right)= & X(g(t)(Y, Z))+Y(g(t)(Z, X))-Z(g(t)(X, Y)) \\
& -g(t)(X,[Y, Z])+g(t)(Y,[Z, X])+g(t)(Z,[X, Y]),
\end{aligned}
$$

(3.2) can be rewritten in form

$$
\begin{aligned}
g(t)(A(X, Y), Z) & =X\left(\frac{1}{2} \frac{\partial^{2} g(t)}{\partial t^{2}}(Y, Z)\right)+Y\left(\frac{1}{2} \frac{\partial^{2} g(t)}{\partial t^{2}}(Z, X)\right) \\
& -Z\left(\frac{1}{2} \frac{\partial^{2} g(t)}{\partial t^{2}}(X, Y)\right)-\frac{1}{2} \frac{\partial^{2} g(t)}{\partial t^{2}}(X,[Y, Z]) \\
& +\frac{1}{2} \frac{\partial^{2} g(t)}{\partial t^{2}}(Y,[Z, X])+\frac{1}{2} \frac{\partial^{2} g(t)}{\partial t^{2}}(Z,[X, Y]) \\
& -\frac{\partial^{2} g(t)}{\partial t^{2}}\left(D_{X} Y, Z\right)-2 \frac{\partial g(t)}{\partial t}(B(X, Y), Z) .
\end{aligned}
$$


By definition of the HGF, we have

$$
\begin{aligned}
g(t)(A(X, Y), Z) & =-X(\operatorname{Ric}(Y, Z))-Y(\operatorname{Ric}(Z, X))+Z(\operatorname{Ric}(X, Y)) \\
& +\operatorname{Ric}(X,[Y, Z])-\operatorname{Ric}(Y,[Z, X])-\operatorname{Ric}(Z,[X, Y]) \\
& +2 \operatorname{Ric}\left(D_{X} Y, Z\right)-2 \frac{\partial g(t)}{\partial t}(B(X, Y), Z) .
\end{aligned}
$$

Note the $A$ is a tensor, we conclude that

$$
\begin{aligned}
g(t)(A(X, Y), Z) & =-\left(D_{X} \operatorname{Ric}(Y, Z)-\left(D_{Y} \operatorname{Ric}\right)(Z, X)\right. \\
& +\left(D_{Z} \operatorname{Ric}\right)(X, Y)-2 \frac{\partial g(t)}{\partial t}(B(X, Y), Z),
\end{aligned}
$$

as claimed.

3.2. Proof of main result 1: Theorem 1.2, Now we return to compute the evolution equation for the curvature tensor. For convenience we need the second order covariant derivative $D_{X, Y}^{2} Z$ defined by

$$
D_{X, Y}^{2} Z:=D_{X} D_{Y} Z-D_{D_{X} Y} Z
$$

from which we have

$$
R(X, Y) Z:=D_{X} D_{Y} Z-D_{Y} D_{X} Z-D_{[X, Y]} Z=D_{X, Y}^{2} Z-D_{Y, X}^{2} Z
$$

Proof of Theorem 1.2 The second derivative of $R(X, Y) Z$ yields

$$
\begin{aligned}
\frac{\partial^{2}}{\partial t^{2}} R(X, Y) Z= & \frac{\partial^{2}}{\partial t^{2}} D_{X}\left(D_{Y} Z\right)+2 \frac{\partial}{\partial t} D_{X}\left(\frac{\partial}{\partial t} D_{Y} Z\right)+D_{X}\left(\frac{\partial^{2}}{\partial t^{2}} D_{Y} Z\right) \\
& -\frac{\partial^{2}}{\partial t^{2}} D_{Y}\left(D_{X} Z\right)-2 \frac{\partial}{\partial t} D_{Y}\left(\frac{\partial}{\partial t} D_{X} Z\right)-D_{Y}\left(\frac{\partial^{2}}{\partial t^{2}} D_{X} Z\right)-\frac{\partial^{2}}{\partial t^{2}} D_{[X, Y]} Z \\
& =A\left(X, D_{Y} Z\right)+2 B(X, B(Y, Z))+D_{X} A(Y, Z)-A\left(Y, D_{X} Z\right) \\
& -2 B(Y, B(X, Z))-D_{Y} A(X, Z)-A\left(D_{X} Y-D_{Y} X, Z\right) \\
& =\left(D_{X} A\right)(Y, Z)-\left(D_{Y} A\right)(X, Z)+2 B(X, B(Y, Z))-2 B(Y, B(X, Z)) .
\end{aligned}
$$

This implies

$$
\begin{aligned}
& \frac{\partial^{2}}{\partial t^{2}} R(X, Y, Z, W) \\
& =\frac{\partial^{2}}{\partial t^{2}}(g(t)(-R(X, Y) Z, W) \\
& =-\frac{\partial^{2} g(t)}{\partial t^{2}}(R(X, Y) Z, W)-g(t)\left(\frac{\partial^{2}}{\partial t^{2}} R(X, Y) Z, W\right) \\
& =2 R i c_{g(t)}(R(X, Y) Z, W)-g(t)\left(\left(D_{X} A\right)(Y, Z), W\right)+g(t)\left(\left(D_{Y} A\right)(X, Z), W\right) \\
& -2 g(t)(B(X, B(Y, Z)), W)+2 g(t)(B(Y, B(X, Z)), W) .
\end{aligned}
$$


Applying Proposition 3.1 we obtain

$$
\begin{aligned}
& g(t)\left(\left(D_{X} A\right)(Y, Z), W\right) \\
& =X(g(t)(A(Y, Z), W))-g(t)\left(A(Y, Z), D_{X} W\right) \\
& -g(t)\left(A\left(D_{X} Y, Z\right), W\right)-g(t)\left(A\left(Y, D_{X} Z\right), W\right) \\
& =X\left(-\left(D_{Y} \operatorname{Ric}_{g(t)}\right)(Z, W)-\left(D_{Z} \operatorname{Ric}_{g(t)}\right)(Y, W)+\left(D_{W} \operatorname{Ric}_{g(t)}\right)(Y, Z)\right. \\
& \left.-2 \frac{\partial g(t)}{\partial t}(B(Y, Z), W)\right)+\left(D_{Y} R i c_{g(t)}\right)\left(Z, D_{X} W\right)+\left(D_{Z} \operatorname{Ric}_{g(t)}\right)\left(Y, D_{X} W\right) \\
& -\left(D_{D_{X} W} R i c_{g(t)}\right)(Y, Z)+2 \frac{\partial g(t)}{\partial t}\left(B(Y, Z), D_{X} W\right) \\
& +\left(D_{D_{X} Y} R_{i c} c_{g(t)}\right)(Z, W)+\left(D_{Z} \operatorname{Ric}_{g(t)}\right)\left(D_{X} Y, W\right) \\
& -\left(D_{W} R_{i c} c_{g(t)}\right)\left(D_{X} Y, Z\right)+2 \frac{\partial g(t)}{\partial t}\left(B\left(D_{X} Y, Z\right), W\right) \\
& +\left(D_{Y} \operatorname{Ric}_{g(t)}\right)\left(D_{X} Z, W\right)+\left(D_{D_{X} Z} R_{i c} c_{g(t)}\right)(Y, W) \\
& -\left(D_{W} \operatorname{Ric}_{g(t)}\right)\left(Y, D_{X} Z\right)+2 \frac{\partial g(t)}{\partial t}\left(B\left(Y, D_{X} Z\right), W\right) \\
& =-\left(D_{X} D_{Y} R i c_{g(t)}-D_{D_{X} Y} R i c_{g(t)}\right)(Z, W)-\left(D_{X} D_{Z} R i c_{g(t)}-D_{D_{X} Z} R i c_{g(t)}\right)(Y, W) \\
& +\left(D_{X} D_{W} R i c_{g(t)}-D_{D_{X} W} R i c_{g(t)}\right)(Y, Z)-2 \frac{\partial g(t)}{\partial t}\left(\left(D_{X} B\right)(Y, Z), W\right) \\
& =-\left(D_{X, Y}^{2} \operatorname{Ric}_{g(t)}\right)(Z, W)-\left(D_{X, Z}^{2} R i c_{g(t)}\right)(Y, W) \\
& +\left(D_{X, W}^{2} R i c_{g(t)}\right)(Y, Z)-2 \frac{\partial g(t)}{\partial t}\left(\left(D_{X} B\right)(Y, Z), W\right) .
\end{aligned}
$$

Interchanging the roles of $X$ and $Y$ yields

$$
\begin{aligned}
& g(t)\left(\left(D_{Y} A\right)(X, Z), W\right) \\
& =-\left(D_{Y, X}^{2} \operatorname{Ric}_{g(t)}\right)(Z, W)-\left(D_{Y, Z}^{2} \operatorname{Ric}_{g(t)}\right)(X, W) \\
& +\left(D_{Y, W}^{2} \operatorname{Ric}_{g(t)}\right)(X, Z)-2 \frac{\partial g(t)}{\partial t}\left(\left(D_{Y} B\right)(X, Z), W\right) .
\end{aligned}
$$

Moreover, we have

$$
\begin{aligned}
& \left(D_{X, Y}^{2} \operatorname{Ric}_{g(t)}\right)(Z, W)-\left(D_{Y, X}^{2} \operatorname{Ric}_{g(t)}\right)(Z, W) \\
& =\left(\left(D_{X, Y}^{2}-D_{Y, X}^{2}\right) R i c_{g(t)}\right)(Z, W) \\
& =\left(R(X, Y) R i c_{g(t)}\right)(Z, W) \\
& =\operatorname{Ric}_{g(t)}(R(X, Y) Z, W)+\operatorname{Ric}_{g(t)}(R(X, Y) W, Z) .
\end{aligned}
$$

Substituting (3.6) with (3.7), (3.8) and (3.9), we get

$$
\begin{aligned}
& \frac{\partial^{2}}{\partial t^{2}} R(X, Y, Z, W) \\
& =-\operatorname{Ric}_{g(t)}(R(X, Y) Z, W)+\operatorname{Ric}_{g(t)}(R(X, Y) W, Z) \\
& +\left(D_{X, Z}^{2} \operatorname{Ric}_{g(t)}\right)(Y, W)-\left(D_{X, W}^{2} \operatorname{Ric}_{g(t)}\right)(Y, Z) \\
& -\left(D_{Y, Z}^{2} \operatorname{Ric}_{g(t)}\right)(X, W)+\left(D_{Y, W}^{2} \operatorname{Ric}_{g(t)}\right)(X, Z) \\
& +2 \frac{\partial g(t)}{\partial t}\left(\left(D_{X} B\right)(Y, Z), W\right)-2 \frac{\partial g(t)}{\partial t}\left(\left(D_{Y} B\right)(X, Z), W\right) \\
& -2 g(t)(B(X, B(Y, Z)), W)+2 g(t)(B(Y, B(X, Z)), W),
\end{aligned}
$$

as claimed. 
3.3. Proof of main result 2: Theorem 1.3, We will show that the right-hand side in the equation (3.10) for the curvature tensor equals the Laplacian of the curvature tensor, up to lower order terms. To this end, we first give the following lemma (See $[\overline{\mathrm{Br}}]$ ) which is independent of any evolution.

Lemma 3.2. Let $X, Y, Z, W$ be arbitrary fields on $M$. Then

$$
\begin{aligned}
& \left(D_{X, Z}^{2} \operatorname{Ric}_{g(t)}\right)(Y, W)-\left(D_{X, W}^{2} \operatorname{Ric}_{g(t)}\right)(Y, Z) \\
& -\left(D_{Y, Z}^{2} \operatorname{Ric}_{g(t)}\right)(X, W)+\left(D_{Y, W}^{2} \operatorname{Ric}_{g(t)}\right)(X, Z) \\
& =(\Delta R)(X, Y, Z, W)+Q(R)(X, Y, Z, W)-\operatorname{Ric}_{g(t)}\left(X, R_{Z, W} Y\right)+\operatorname{Ric}_{g(t)}\left(Y, R_{Z, W} X\right),
\end{aligned}
$$

where $Q(R):=R^{2}+R^{\#}$ is a curvature tensor satisfying the first Bianchi identity, and given a local orthonormal basis $\left\{e_{1}, \ldots, e_{n}\right\}, R^{2}$ and $R^{\#}$ are difined by (1.6) in Theorem 1.3

Proof. For the orthonormal basis $\left\{e_{1}, \ldots, e_{n}\right\}$, it is easy to show that

$$
\begin{aligned}
& \left(D_{X, Z}^{2} \operatorname{Ric}_{g(t)}\right)(Y, W)=\sum_{k=1}^{n}\left(D_{X, Z}^{2} R\right)\left(e_{k}, Y, e_{k}, W\right), \\
& \left(D_{X, W}^{2} R i c_{g(t)}\right)(Y, Z)=\sum_{k=1}^{n}\left(D_{X, W}^{2} R\right)\left(e_{k}, Y, e_{k}, Z\right) .
\end{aligned}
$$

Using the second Bianchi identity, by a direct computation we obtain

$$
\left(D_{X, Z}^{2} R\right)\left(e_{k}, Y, e_{k}, W\right)-\left(D_{X, W}^{2} R\right)\left(e_{k}, Y, e_{k}, Z\right)=\left(D_{X, e_{k}}^{2} R\right)\left(e_{k}, Y, Z, W\right) .
$$

Thus we have

$$
\left(D_{X, Z}^{2} \operatorname{Ric}_{g(t)}\right)(Y, W)-\left(D_{X, W}^{2} \operatorname{Ric}_{g(t)}\right)(Y, Z)=\sum_{k=1}^{n}\left(D_{X, e_{k}}^{2} R\right)\left(e_{k}, Y, Z, W\right),
$$

and

$$
\left(D_{Y, Z}^{2} R i c_{g(t)}\right)(X, W)-\left(D_{Y, W}^{2} R i c_{g(t)}\right)(X, Z)=\sum_{k=1}^{n}\left(D_{Y, e_{k}}^{2} R\right)\left(e_{k}, X, Z, W\right) .
$$

Therefore, (3.14) and (3.15) yield

$$
\begin{aligned}
I & :=\left(D_{X, Z}^{2} \operatorname{Ric}_{g(t)}\right)(Y, W)-\left(D_{X, W}^{2} \operatorname{Ric}_{g(t)}\right)(Y, Z) \\
- & \left(D_{Y, Z}^{2} \operatorname{Ric}_{g(t)}\right)(X, W)+\left(D_{Y, W}^{2} R i c_{g(t)}\right)(X, Z) \\
& \left.=\sum_{k=1}^{n}\left(D_{X, e_{k}}^{2} R\right)\left(e_{k}, Y, Z, W\right)-\left(D_{Y, e_{k}}^{2} R\right)\left(e_{k}, X, Z, W\right)\right) .
\end{aligned}
$$

Now we consider to put $Q(R)$ into 3.16 . Note that

$$
\begin{aligned}
& \sum_{k=1}^{n}\left(D_{X, e_{k}}^{2} R-D_{e_{k}, X}^{2} R\right)\left(e_{k}, Y, Z, W\right) \\
& =\sum_{k, l=1}^{n}\left(R\left(X, e_{k}, e_{k}, e_{l}\right) R\left(e_{l}, Y, Z, W\right)+R\left(X, e_{k}, Y, e_{l}\right) R\left(e_{k}, e_{l}, Z, W\right)\right. \\
& \left.\quad+R\left(X, e_{k}, Z, e_{l}\right) R\left(e_{k}, Y, e_{l}, W\right)+R\left(X, e_{k}, W, e_{l}\right) R\left(e_{k}, Y, Z, e_{l}\right)\right)
\end{aligned}
$$


and

$$
\begin{aligned}
& \sum_{k=1}^{n}\left(D_{Y, e_{k}}^{2} R-D_{e_{k}, Y}^{2} R\right)\left(e_{k}, X, Z, W\right) \\
& =\sum_{k, l=1}^{n}\left(R\left(Y, e_{k}, e_{k}, e_{l}\right) R\left(e_{l}, X, Z, W\right)+R\left(Y, e_{k}, X, e_{l}\right) R\left(e_{k}, e_{l}, Z, W\right)\right. \\
& \left.\quad+R\left(Y, e_{k}, Z, e_{l}\right) R\left(e_{k}, X, e_{l}, W\right)+R\left(Y, e_{k}, W, e_{l}\right) R\left(e_{k}, X, Z, e_{l}\right)\right)
\end{aligned}
$$

we have

$$
\begin{aligned}
& \sum_{k=1}^{n}\left(\left(D_{X, e_{k}}^{2} R-D_{e_{k}, X}^{2} R\right)\left(e_{k}, Y, Z, W\right)-\left(D_{Y, e_{k}}^{2} R-D_{e_{k}, Y}^{2} R\right)\left(e_{k}, X, Z, W\right)\right) \\
& =\sum_{l=1}\left(R i c\left(X, e_{l}\right) R\left(e_{l}, Y, Z, W\right)-\operatorname{Ric}\left(Y, e_{l}\right) R\left(e_{l}, X, Z, W\right)\right) \\
& +\sum_{k, l=1}^{n}\left(R\left(X, e_{k}, Y, e_{l}\right)-R\left(Y, e_{k}, X, e_{l}\right)\right) R\left(e_{k}, e_{l}, Z, W\right) \\
& +2 \sum_{k, l=1}^{n}\left(R\left(X, e_{k}, Z, e_{l}\right) R\left(e_{k}, Y, e_{l}, W\right)-R\left(X, e_{k}, W, e_{l}\right) R\left(Y, e_{k}, Z, e_{l}\right)\right)
\end{aligned}
$$

By definitions of $R^{2}$ and $R^{\#}$, together with the first Bianchi identity

$$
R\left(X, e_{k}, Y, e_{l}\right)-R\left(Y, e_{k}, X, e_{l}\right)=R\left(X, Y, e_{k}, e_{l}\right),
$$

(3.19) can be reduced as

$$
\begin{aligned}
& \sum_{k=1}^{n}\left(\left(D_{X, e_{k}}^{2} R-D_{e_{k}, X}^{2} R\right)\left(e_{k}, Y, Z, W\right)-\left(D_{Y, e_{k}}^{2} R-D_{e_{k}, Y}^{2} R\right)\left(e_{k}, X, Z, W\right)\right) \\
& =-\operatorname{Ric}\left(X, R_{Z, W} Y\right)+\operatorname{Ric}\left(Y, R_{Z, W} X\right)+\left(R^{2}+R^{\#}\right)(X, Y, Z, W) .
\end{aligned}
$$

Hence, from (3.16) and (3.20) we have

$$
\begin{aligned}
& \left.I=Q(R)(X, Y, Z, W)+\sum_{k=1}^{n}\left(D_{e_{k}, X}^{2} R\right)\left(e_{k}, Y, Z, W\right)-\left(D_{e_{k}, Y}^{2} R\right)\left(e_{k}, X, Z, W\right)\right) \\
& -\operatorname{Ric}\left(X, R_{Z, W} Y\right)+\operatorname{Ric}\left(Y, R_{Z, W} X\right) .
\end{aligned}
$$

Next we consider to put $\Delta R$ into (3.20). Similar to (3.13), we have

$$
\sum_{k=1}^{n}\left(D_{e_{k}, e_{k}}^{2} R\right)(X, Y, Z, W)=\sum_{k=1}^{n}\left(\left(D_{e_{k}, X}^{2} R\right)\left(e_{k}, Y, Z, W\right)-\left(D_{e_{k}, Y}^{2} R\right)\left(e_{k}, X, Z, W\right)\right) .
$$

Moreover, we know

$$
\Delta R=\sum_{k=1}^{n} D_{e_{k}, e_{k}}^{2} R
$$

Putting these facts together, (3.21) arrives at the desired equation. 
Proof of Theorem 1.3 By Theorem 1.2 and Lemma 3.2, we obtain the following wavecharacter equation for curvature tensor

$$
\begin{aligned}
\frac{\partial^{2}}{\partial t^{2}} R(X, Y, Z, W)= & (\Delta R)(X, Y, Z, W)+Q(R)(X, Y, Z, W) \\
& -\operatorname{Ric}_{g(t)}\left(X, R_{Z, W} Y\right)+\operatorname{Ric}_{g(t)}\left(Y, R_{Z, W} X\right) \\
+ & R i c_{g(t)}\left(Z, R_{X, Y} W\right)-\operatorname{Ric}_{g(t)}\left(W, R_{X, Y} Z\right) \\
+ & 2 \frac{\partial g(t)}{\partial t}\left(\left(D_{X} B\right)(Y, Z), W\right)-2 \frac{\partial g(t)}{\partial t}\left(\left(D_{Y} B\right)(X, Z), W\right) \\
- & 2 g(t)(B(X, B(Y, Z)), W)+2 g(t)(B(Y, B(X, Z)), W) .
\end{aligned}
$$

3.4. Proof of main result 3: Theorem 1.4, Notice that the hyperbolic geometric flow is an evolution equation on the metric $g_{i j}(t)$. The evolution for the metric is not only implies a nonlinear wave equation for the Riemannian curvature tensor, but also for the Ricci curvature tensor and the scalar curvature. This result (i.e.Theorems 1.4) is stated in the introduction. Now we give its proof.

Proof of Theorem [1.4 Similar to (3.12), we have

$$
\begin{aligned}
\left(\Delta \operatorname{Ric}_{g(t)}\right)(X, Y) & =\left(\sum_{j=1}^{n}\left(D_{e_{j}, e_{j}}^{2} R i c\right)\right)(X, Y) \\
& =\sum_{i, j=1}^{n}\left(D_{e_{j}, e_{j}}^{2} R\right)\left(X, e_{i}, Y, e_{i}\right) \\
& =\sum_{i=1}^{n}(\Delta R)\left(X, e_{i}, Y, e_{i}\right) .
\end{aligned}
$$

By definition of $Q(R)$, we have

$$
\begin{aligned}
\sum_{i=1}^{n} Q(R)\left(X, e_{i}, Y, e_{i}\right) & =\sum_{i, j, k=1}\left(R\left(X, e_{i}, e_{j}, e_{k}\right) R\left(Y, e_{i}, e_{j}, e_{k}\right)\right. \\
& +2 R\left(X, e_{j}, Y, e_{k}\right) R\left(e_{i}, e_{j}, e_{i}, e_{k}\right) \\
& \left.-2 R\left(e_{i}, e_{j}, Y, e_{k}\right) R\left(X, e_{j}, e_{i}, e_{k}\right)\right) .
\end{aligned}
$$

Using the first Bianchi identity, we obtain

$$
\begin{aligned}
& -2 \sum_{i, j, k=1} R\left(e_{i}, e_{j}, Y, e_{k}\right) R\left(X, e_{j}, e_{i}, e_{k}\right) \\
& =-\sum_{i, j, k=1} R\left(X, e_{j}, e_{i}, e_{k}\right)\left(R\left(Y, e_{k}, e_{i}, e_{j},\right)-R\left(Y, e_{i}, e_{k}, e_{j}\right)\right) \\
& =-\sum_{i, j, k=1} R\left(X, e_{j}, e_{i}, e_{k}\right) R\left(Y, e_{j}, e_{i}, e_{k}\right) \\
& =-\sum_{i, j, k=1} R\left(X, e_{i}, e_{j}, e_{k}\right) R\left(Y, e_{i}, e_{j}, e_{k}\right) .
\end{aligned}
$$


Hence (3.24) can be reduced as

$$
\begin{aligned}
\sum_{i=1}^{n} Q(R)\left(X, e_{i}, Y, e_{i}\right) & =2 \sum_{i, j, k=1} R\left(X, e_{j}, Y, e_{k}\right) R\left(e_{i}, e_{j}, e_{i}, e_{k}\right) \\
& =2 \sum_{j, k=1} R\left(X, e_{j}, Y, e_{k}\right) R i c_{g(t)}\left(e_{j}, e_{k}\right) .
\end{aligned}
$$

Moreover, we have

$$
\begin{aligned}
& \sum_{i=1}^{n}\left[-R i c_{g(t)}\left(X, R_{Y, e_{i}} e_{i}\right)+R i c_{g(t)}\left(e_{i}, R_{Y, e_{i}} X\right)\right. \\
& \left.+\operatorname{Ric}_{g(t)}\left(Y, R_{X, e_{i}} e_{i}\right)-\operatorname{Ric}_{g(t)}\left(e_{i}, R_{X, e_{i}} Y\right)\right] \\
& =\sum_{i, j=1}^{n}\left[-R\left(Y, e_{i}, e_{i}, e_{j}\right) R i c_{g(t)}\left(X, e_{j}\right)+R\left(Y, e_{i}, X, e_{j}\right) R i c_{g(t)}\left(e_{i}, e_{j}\right)\right. \\
& \left.+R\left(X, e_{i}, e_{i}, e_{j}\right) R i c_{g(t)}\left(Y, e_{j}\right)-R\left(X, e_{i}, Y, e_{j}\right) R i c_{g(t)}\left(e_{i}, e_{j}\right)\right] \\
& =\sum_{i, j=1}^{n}\left[R i c_{g(t)}\left(Y, e_{j}\right) \operatorname{Ric} c_{g(t)}\left(X, e_{j}\right)-\operatorname{Ric}_{g(t)}\left(X, e_{j}\right) \operatorname{Ric} c_{g(t)}\left(Y, e_{j}\right)\right] \\
& =0
\end{aligned}
$$

Using Theorem 1.3, or by (3.22), together with (3.23), (3.25) and (3.26), we get

$$
\begin{aligned}
& \frac{\partial^{2}}{\partial t^{2}} \operatorname{Ric}_{g(t)}(X, Y)=\sum_{i=1}^{n} \frac{\partial^{2}}{\partial t^{2}} R\left(X, e_{i}, Y, e_{i}\right) \\
& =\left(\Delta R i c_{g(t)}\right)(X, Y)+2 \sum_{j, k=1} R\left(X, e_{j}, Y, e_{k}\right) R i c_{g(t)}\left(e_{j}, e_{k}\right) \\
& \left.+2 \sum_{i=1}^{n}\left[\frac{\partial g(t)}{\partial t}\left(\left(D_{X} B\right)\left(e_{i}, Y\right), e_{i}\right)\right)-\frac{\partial g(t)}{\partial t}\left(\left(D_{e_{i}} B\right)(X, Y), e_{i}\right)\right] \\
& -2 \sum_{i=1}^{n}\left[g(t)\left(B\left(X, B\left(e_{i}, Y\right)\right), e_{i}\right)-g(t)\left(B\left(e_{i}, B(X, Y)\right), e_{i}\right)\right],
\end{aligned}
$$

as the first claimed equality (1.7).

As for the second assertion (1.8), note that

$$
\begin{aligned}
& \frac{\partial^{2}}{\partial t^{2}} \operatorname{Scal}_{g(t)}=\sum_{i=1}^{n} \frac{\partial^{2}}{\partial t^{2}} \operatorname{Ric}_{g(t)}\left(e_{i}, e_{i}\right), \\
& \sum_{i=1}^{n}\left(\Delta R i c_{g(t)}\right)\left(e_{i}, e_{i}\right)=\Delta s c a l_{g(t)}, \\
& \sum_{i, j, k=1} R\left(e_{i}, e_{j}, e_{i}, e_{k}\right) R i c_{g(t)}\left(e_{j}, e_{k}\right)=\sum_{j, k=1} \operatorname{Ric}_{g(t)}\left(e_{j}, e_{k}\right) R i c_{g(t)}\left(e_{j}, e_{k}\right)=\left|R i c_{g(t)}\right|^{2},
\end{aligned}
$$


it follows from (3.27) that

$$
\begin{aligned}
\frac{\partial^{2}}{\partial t^{2}} S \operatorname{cal} l_{g(t)}= & \Delta S c a l_{g(t)}+2\left|R i c_{g(t)}\right|^{2} \\
& \left.+2 \sum_{i, j=1}^{n}\left[\frac{\partial g(t)}{\partial t}\left(\left(D_{e_{j}} B\right)\left(e_{i}, e_{j}\right), e_{i}\right)\right)-\frac{\partial g(t)}{\partial t}\left(\left(D_{e_{i}} B\right)\left(e_{j}, e_{j}\right), e_{i}\right)\right] \\
& -2 \sum_{i, j=1}^{n}\left[g(t)\left(B\left(e_{j}, B\left(e_{i}, e_{j}\right)\right), e_{i}\right)-g(t)\left(B\left(e_{i}, B\left(e_{j}, e_{j}\right)\right), e_{i}\right)\right],
\end{aligned}
$$

as claimed.

\section{Curvature blow-up at finite-TIMe singularities}

In this section, we consider a maximal solution to the hyperbolic geometric flow which is defined on a finite interval $[0, T)$. Similar to the result in Hamilton's paper $([\mathrm{Ha}]$, Theorem 14.1), we show that such a solution must have unbounded curvature. The proof is due to C. Udriste's approach (See [Ud], Theorem 6.1).

Theorem 4.1. Let $M$ be a compact manifold, and let $g(t), t \in[0, T)$, be a maximal solution to the HGF (2.1) on M. Moreover, suppose that $T<\infty$. Then

$$
\limsup _{t \rightarrow T}\left(\sup _{M}\left|R i c_{g(t)}\right|\right)=\infty .
$$

Proof. We argue by contradiction. Assuming the Ricci tensor of $g(t)$ is uniformly bounded for all $t \in[0, T)$, that is, there is a positive constant $m$ such that $\left|R i c_{g(t)}\right| \leq m, t \in[0, T)$. So the solution $g(t)$ can be extended to a larger time interval $[0, T+\varepsilon)$, where $\varepsilon$ is a arbitrary small number. Indeed, by (2.1), we have the relations

$$
\begin{aligned}
& \frac{\partial g}{\partial t}(t)=\frac{\partial g}{\partial t}(0)-2 \int_{0}^{t} \operatorname{Ric}(s, x) d s \\
& g(t)=g(0)+t \frac{\partial g}{\partial t}(0)-2 \int_{0}^{t} \int_{0}^{u} \operatorname{Ric}(s, x) d s d u, t \in[0, T)
\end{aligned}
$$

which imply

$$
g\left(t_{1}\right)-g\left(t_{2}\right)=\left(t_{1}-t_{2}\right) \frac{\partial g}{\partial t}(0)-2 \int_{t_{1}}^{t_{2}} \int_{0}^{u} \operatorname{Ric}(s, x) d s d u, t_{1}, t_{2} \in[0, T)
$$

Hence we obtain

$$
\left|g\left(t_{1}\right)-g\left(t_{2}\right)\right|=\left(\left|\frac{\partial g}{\partial t}(0)\right|+2 m T\right)\left|t_{1}-t_{2}\right|
$$

The Cauchy Criterion shows that $\lim _{t \rightarrow T} g(t)$ exists, while $\lim _{t \rightarrow T} \frac{\partial g}{\partial t}(t)$ and $\lim _{t \rightarrow T} \frac{\partial^{2} g}{\partial t^{2}}(t)$ exist since $\lim _{t \rightarrow T} \operatorname{Ric}(t, x)$ exists (See Definition 2.1). Consequently, $g(t), t \in[0, T]$ is the solution to a HGF.

In this case, $g(t)$ may be extended from being a smooth solution on $[0, T)$ to a smooth solution on $[0, T]$. Then we take $g(T), \frac{\partial g}{\partial t}(T)$ to be an initial metric in a short-time existence theorem in order to extend the solution to a HGF for $t \in[0, T+\varepsilon)$. This contradicts the assumption that $[0, T)$ is a maximal time interval. Therefore, we complete the proof. 


\section{EXPECTED PROBLEMS}

To conclude this paper, we present some expected problems. Now we have the global forms of evolution equations (1.4), (1.5), (1.7) and (1.8) along the HGF (2.1), so in the future we are interested in the following problems:

1. For fixed $(p, t) \in M \times[0, T]$, we denote by $K_{\max (p, t)} / K_{\min }(p, t)$ the maximum /minimum sectional curvature of $g(t)$ at the point $p$. Moreover, for abbreviation, we define

$$
K_{\max }(t)=\sup _{p \in M} K_{\max }(p, t), K_{\min }(t)=\inf _{p \in M} K_{\min }(p, t) .
$$

Let $\left\{t_{k}\right\}$ be a sequence of times such that $\lim _{k \rightarrow \infty} t_{k}=T$ and $K_{\max }\left(t_{k}\right) \geq \frac{1}{2} \sup _{t \in\left[0, t_{k}\right]} K_{\max }(t)$ for all $k$. Then by Theorem 4.1, does the following relation hold

$$
\begin{aligned}
& \limsup _{k \rightarrow \infty} \frac{K_{\min }\left(t_{k}\right)}{K_{\max }\left(t_{k}\right)}<1, \text { or } \\
& \lim _{t \rightarrow T} \frac{K_{\min }(t)}{K_{\max }(t)}=1 \text { ? }
\end{aligned}
$$

2. (Preserved curvature conditions by the $H G F$ ) We know that if we want to study the global properties of HGF, then it is important to find curvature conditions that are preserved under the evolution. How to develop such techniques? For instance, suppose $M$ is compact manifold, and let $g(t), t \in[0, T)$, be a solution to HGF on $M$, and consider a appropriate $\operatorname{ODE}(*) \frac{d^{2}}{d t^{2}} R(t)=Q(R(t))+($ certain term $)$. Can we claim that nonnegative isometric curvature $([\overline{\mathrm{Br}}])$ is preserved by the $\mathrm{ODE}(*)$ ?

Acknowledgments. I am very grateful to Professor Kefeng Liu , as my advisor, who has encouraged me to learn the newest developments from leading geometric experts and has been offering me tremendous information and constructive suggestions. I would like to thank Professor Hong-Wei Xu for helpful discussions on the course Ricci Flow and the Sphere Theorem by S.Brendle [B] $]$.

\section{REFERENCES}

[BLN] Bennett Chow, Peng Lu and Lei Ni, Harmilton's Ricci Flow, American Mathematical Society and Science Press, 2006.

[Br] S. Brendle, Ricci Flow and the Sphere Theorem, Grad. Studies in Math., 111, AMS., 2010.

[BS] S.Brendle and R.Schoen, Manifolds with 1/4-pinched curvature are space forms, J. Amer.Math.Sco., 2009,200:1-13.

[Br1] S. Brendle, Convergence of the Yamabe flow for arbitrary initial energy, J. Differential Geom., 2005, 69:217-278.

[Br2] S. Brendle, Convergence of the Yamabe flow in dimension 6 and higher, Invent. Math., 2007, 170:541-576.

[Ch1] B. Chow, The Yamabe fow on locally conformally flat manifolds with positive Ricci curvature, Comm. Pure Appl. Math., 1992, 65:1003-1014.

[DKL1] Wen-Rong Dai, De-Xing Kong and Kefeng Liu, Hyperbolic geometric flow (I): short-time existence and nonlinear stability, Pure and Applied Mathematics Quarterly, 2010, 6(2):331-359 (Special Issue: In honor of Michael Atiyah and Isadore Singer).

[DKL2] Wen-Rong Dai, De-Xing Kong and Kefeng Liu, Dissipative hyperbolic geometric flow, Asia J Math, 2008, 12: 345-364.

[De] D. De Turk, Deforming metrics in the derection of their Ricci tensors, J. Diffrential Geom., 1983, 18:157162. 
[Ha] R. Hamilton,Three-manifolds with positive Ricci curvature, J. Differential Geom., 1982, 17:255-306.

[He] Chun-Lei He, Exact solutions for Einstein's hyperbolic geometric flow, Communications in Theoretical Physics, 2008, 50: 1331-1553

[Jo] J. Jost, Riemannian Geometry and Geometric Analysis, 6th ed., Springer, 2011.

[KL] De-Xing Kong and Kefeng Liu, Wave character of metrics and hyperbolic geometric flow, J. Math. Phys, 2007,48:103508-1-103508-14.

[KLX] De-Xing Kong, Kefeng Liu and De-Liang Xu, The hyperbolic geometric flow on Riemann surfaces, Communications in Partial Differntial Equations, 2009, 34: 553-580.

[KLW] De-Xing Kong, Kefeng Liu and Y Z. Wang, Life-span of classical solutions to hyperbolic geometric flow in two space variables with slow decay initial data, Communications in Partial Differential Equations, 2011, 36:162-184.

[Ko] De-Xing Kong, Hyperbolic Geometric Flow, The Proceedings of ICCM 2007, Vol. II. Beijing: Higher Educationial Press, 2007: 95-110.

[Li] Liu K., Hyperbolic geometric flow, Lecture at International Conference of Elliptic and Parabolic Differential Equations, Hangzhou, August 20, 2007. Available at preprint webpage of Center of Mathematical Science, Zhejiang University

[MR] A.Milgram and P.Rosenblum, Harmonic forms and heat conduction, I: closed Riemannian manifolds, Proc. Nat. Acad. Sci., 1951, 37:180-184.

[To] P.Topping, Lectures on the Ricci Flow, LMS Lecture Notes 325, London Math. Society and Cambridge University Press, 2006.

[Re] R. Ye, Global existence and convergence of Yamabe flow, J.Differential Geom., 1994, 39:35-50.

[SS] H. Schwetlick,M. Struwe, Convergence of the Yamabe flow for "large" energies, J. Reine Angew. Math., 2003, 562:59-100.

[SY] R. Schoen and S.T. Yau, Lectures on Differential Geometry, International Press, Boston, 1994.

[Ud] C.Udriste, Riemann flow and Riemann wave via bialternate product Riemannian metric, arXiv:1112.4279 $\mathrm{l}, 2011$.

[Zh] Xi-Ping Zhu, Lectures on Mean Curvature Flows, Studies in Advanced Mathematics 32. AMS/IP, 2002.

Center of Mathematical Sciences, Zhejiang University, Hangzhou,Zhejiang, 310027, P. R. China.

E-mail address: weijunlu2008@126.com 